\title{
Styrene-Divinylbenzene Copolymers. Construction of Porosity in Styrene Divinylbenzene Matrices
}

\author{
W. L. SEDEREL and G. J. DE JONG,* AKZO-Zout Chemie Research, \\ Hengelo, The Netherlands, and Polymer Division, Department of \\ Chemical Technology, Twente University of Technology, \\ Enschede, The Netherlands
}

\begin{abstract}
Synopsis
Experimental data are presented describing the formation of porosity in styrene-divinylbenzene copolymers as a function of the organic components present during the suspension polymerization. The reaction system contains a mixture of diluents such as toluene and hexane, which results in matrices that differ significantly in pore structure from the porous resins so far known. From these data a model of the physical structure is proposed.
\end{abstract}

\section{INTRODUCTION}

During the last ten years, there has been an increasing interest in the styrene-divinylbenzene copolymer as a porous starting material for a number of ion exchangers. According to Seidl, ${ }^{1}$ there are three methods to prepare porous matrices:

1. By addition of a solvating diluent $t^{2,3,4}$ such as toluene and dichloroethane to the polymerizing mixture. We propose to name this type of copolymer a PS type (porous by solvent). It is characterized by a relatively low pore volume (up to about $0.8 \mathrm{ml} / \mathrm{g}$ ), a considerable internal surface $\left(50-500 \mathrm{~m}^{2} / \mathrm{g}\right)$, and a small average pore diameter.

2. By addition of a nonsolvating diluent $t^{4,5,6}$ such as $n$-heptane and $n$ butyl alcohol, resulting in a PP type copolymer (porous by precipitator). It is characterized by a large pore volume $(0.6-2.0 \mathrm{ml} / \mathrm{g})$, an internal surface varying from 10 to $100 \mathrm{~m}^{2} / \mathrm{g}$, and a relatively large average pore diameter.

3 . By addition of a linear polymer, ${ }^{7,8,9}$ e.g., polystyrene, which gives a PM type copolymer (porous by macromolecular material). The characterization is a pore volume up to about $0.5 \mathrm{ml} / \mathrm{g}$, internal surface 0-10 $\mathrm{m}^{2} / \mathrm{g}$, and a large average pore diameter.

The given characterizations are dependent on the concentration of the crosslinking agent, concentration of the diluents, and the reaction temperature. The values are only meant as tendencies.

* Present address: AKZO-Zout Chemie Research, Hengelo, The Netherlands. 
By adding two diluents at the same time to the polymerizing mixture, we get three new types of matrices, i.c., the PPS, PMS, and PMP type. Evidently these matrices are of interest when we succeed in combining the favorite properties of one type with those of the other one. This results in matrices having a different pore structure and pore distribution compared with the thus far known copolymers.

\section{NOMENCLATURE}

As described by Kun and Kunin, ${ }^{10}$ the availability of what they call macroreticular structures raises a problem in nomenclature. We want to state that there is no reason to deviate from the normal definitions used in the field of sorbents and catalysts. Therefore, we will not use the terms micro- and macroreticular resins. Instead, we shall use the conventional definition of porosity based on the mechanism of pore structure formation as given in Figure 1.11

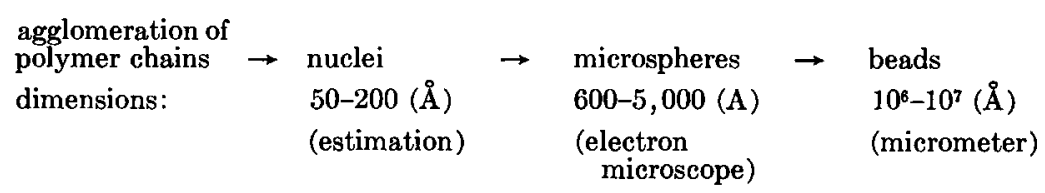

Fig. 1.

1. Gel porosity: exists only in the swollen state of a macromolecular network, e.g., an ion exchange matrix swollen in water or a crosslinked matrix swollen in an organic solvent.

2. Macroporosity: due to the pores among the microspheres and their agglomerations, the pore diameters exceed $250 \AA$.

3. Microporosity: due to the pores present within the microspheres, among the nuclei and their agglomerations. The pores have diameters varying from some tens to some hundreds of angströms and form the internal surface.

The pores are classified in the usual manner for sorbents and catalysts: (a) micropores, $<50 \AA$; (b) transitional pores, $50-500 \AA$; (c) macropores, $>500 \AA$. Table I demonstrates the relation between porosity and pore size.

TABLE I

Relation of Porosity to Pore Size

\begin{tabular}{cccc} 
& \multicolumn{3}{c}{ Pore diameter, $\AA$} \\
\cline { 3 - 4 } Gel porosity & Macroporosity & Microporosity \\
\hline $\begin{array}{c}\text { No pores in } \\
\text { dry state }\end{array}$ & $\stackrel{500}{\leftarrow}$ & 250 & 50 \\
- & macro & transitional & $\overrightarrow{\text { micro }}$ \\
\hline
\end{tabular}




\section{EXPERIMENTAL}

\section{Preparation of Matrices}

The suspension polymerizations were run in a standard suspension polymcrization apparatus, consisting of a 3-liter, four-nccked, round-bottomed flask fitted with a mechanical stirrer, a thermometer, and a reflux condenser with a nitrogen gas inlet tube at the top. A solution of styrene, technicalgrade divinylbenzene (containing $\pm 60 \%$ divinylbenzene), diluents, and benzoylperoxide (containing $75 \%$ benzoylperoxide) with a total weight of $300 \mathrm{~g}$ was added to a solution of bentonite $(3 \mathrm{~g})$, gelatin $(3 \mathrm{~g})$, and $\mathrm{NaCl}$ $(3 \mathrm{~g})$ in $1200 \mathrm{ml}$ water. The mixture was agitated until the organic components were dispersed as fine droplets and then heated at $80^{\circ} \mathrm{C}$ for about $16 \mathrm{hr}$ in a thermostated oil bath. The resulting beads were collected and sieved, and the fractions were washed with acetone and ethanol and dried overnight in air.

When a linear polymer was used as diluent, the beads were collected and then extracted with dichloromethane in a Soxhlet apparatus, followed by washing with a mixture of dichloromethane and ethanol and a portion of ethanol only. The beads were then sieved and dried overnight.

\section{Methods of Determining Pore Structure}

Pore structures of the copolymers were characterized by apparent densities, total porosities, average pore sizes, pore size distributions, and surface area determinations.

The apparent densities were measured in a measuring cylinder. Total porosities were determined by a mercury penetrometer (type Carlo Ponti).

TABLE II

Experimental Results for PPS Copolymerss

\begin{tabular}{cccccccc}
\hline $\begin{array}{c}\text { Exp. } \\
\text { no. }\end{array}$ & $\begin{array}{c}\text { DVB, } \\
\%\end{array}$ & $\begin{array}{c}\text { Hexane, } \\
\%\end{array}$ & $\begin{array}{c}\text { Toluene, } \\
\%\end{array}$ & $\begin{array}{c}\rho_{0}, \\
\mathrm{~g} / \mathrm{cm}^{3}\end{array}$ & $\begin{array}{c}P V, \\
\mathrm{ml} / \mathrm{g}\end{array}$ & $\begin{array}{c}S_{\mathrm{BET}}, \\
\mathrm{m}^{2} / \mathrm{g}\end{array}$ & $\bar{D}, \AA^{\mathrm{b}}$ \\
\hline $01^{\mathrm{c}}$ & 15 & $100^{\mathrm{c}}$ & - & $\mathbf{0 . 4 0}$ & 1.62 & - & - \\
02 & 15 & 80 & 20 & $\mathbf{0 . 4 3}$ & 1.24 & - & - \\
03 & 15 & 60 & 40 & $\mathbf{0 . 6 4}$ & 0.57 & - & - \\
04 & 15 & - & 100 & $\mathbf{0 . 9 8}$ & 0.10 & - & - \\
05 & 10 & 150 & 15 & 0.45 & 1.48 & 22 & 2700 \\
06 & 10 & 150 & 20 & 0.55 & 0.80 & 32 & 1000 \\
07 & 10 & 150 & 30 & 0.76 & - & - & - \\
08 & 10 & 150 & 40 & 0.84 & - & - & - \\
09 & 35 & 150 & - & 0.34 & 1.9 & 114 & 700 \\
10 & 35 & 150 & 15 & 0.37 & 1.6 & 167 & 380 \\
11 & 35 & 150 & 60 & 0.32 & 1.2 & 229 & 210 \\
12 & 35 & 150 & 100 & 0.47 & 0.95 & 296 & 120 \\
\hline
\end{tabular}

- DVB $=$ Divinylbenzene; $\rho_{0}=$ apparent density; $P V=$ pore volume; $S_{\mathrm{BET}}=$ internal surface; $D=$ average pore diameter.

b Calculated from $\bar{D}=\left(4 P V / S_{\mathrm{BET}}\right) \times 10^{4}$ (cylindrical model assumed).

c All \% are weight percentages, except for exp. 01-04. 
TABLE III

Experimental Results for PMS and PPS Copolymers

\begin{tabular}{rrrrrrrr}
\hline $\begin{array}{c}\text { Exp. } \\
\text { no. }\end{array}$ & $\begin{array}{c}\text { DVB, } \\
\%\end{array}$ & $\begin{array}{c}\text { Hexane, } \\
\%\end{array}$ & $\begin{array}{c}\text { Toluene, } \\
\%\end{array}$ & $\begin{array}{c}L P, \\
\%\end{array}$ & $M_{w}$ & $\begin{array}{c}\rho_{0}, \\
{\mathrm{~g} / \mathrm{cm}^{3}}^{3}\end{array}$ & $\begin{array}{c}P V, \\
\mathrm{ml} / \mathrm{g}\end{array}$ \\
\hline 13 & 16 & - & - & 15 & 33,000 & $\mathbf{0 . 9 2}$ & 0.08 \\
14 & 16 & - & 20 & 15 & 33,000 & 0.80 & 0.21 \\
15 & 16 & - & 60 & 15 & 33,000 & 0.75 & 0.32 \\
16 & 16 & - & 100 & 15 & 33,000 & 0.77 & 0.25 \\
17 & 25 & - & - & 15 & 33,000 & 0.79 & 0.13 \\
18 & 25 & - & 20 & 15 & 33,000 & 0.72 & 0.28 \\
19 & 25 & - & 60 & 15 & 33,000 & 0.67 & 0.41 \\
20 & 25 & - & 100 & 15 & 33,000 & 0.56 & 0.58 \\
21 & 8 & - & 20 & 15 & 33,000 & 0.91 & 0.04 \\
22 & 8 & - & 60 & 15 & 33,000 & 0.90 & 0.03 \\
23 & 8 & - & 100 & 15 & 33,000 & 0.93 & 0.05 \\
24 & 8 & - & 100 & 30 & 33,000 & 0.90 & 0.03 \\
25 & 16 & - & 100 & 30 & 33,000 & 0.62 & 0.40 \\
26 & 25 & - & 100 & 30 & 33,000 & 0.53 & 0.77 \\
27 & 25 & 30 & - & 5 & 33,000 & 0.76 & 0.31 \\
28 & 25 & 80 & - & 5 & 33,000 & 0.69 & 0.45 \\
29 & 25 & 120 & - & 5 & 33,000 & 0.31 & 2.0 \\
30 & 10 & 60 & - & 2 & 140,000 & 0.64 & 0.40 \\
31 & 10 & 60 & - & 4 & 140,000 & 0.67 & 0.58 \\
32 & 10 & 60 & - & 6 & 140,000 & 0.60 & 0.60 \\
33 & 10 & 60 & - & 8 & 140,000 & 0.58 & 0.72 \\
34 & 10 & 60 & - & 15 & 140,000 & 0.47 & 1.0 \\
35 & 10 & 60 & - & 15 & 33,000 & 0.59 & 0.63 \\
\hline
\end{tabular}

all percentages are weight- $\%$; LP $=$ linear polymer; $M_{w}=$ weight-average molecular weight.

For the larger pores, pore size distributions can be correlated with distributions obtained by electron micrographs. The surface areas were obtained by nitrogen adsorption-desorption isotherms using the Brunauer, Emmet, and Teller (B.E.T.) method. ${ }^{12}$

Table II shows experimental results for PPS copolymers; Table III, experimental results for PMS and PPS copolymers.

\section{RESULTS}

\section{PPS Resin}

It must be remembered that porous matrices are built when the amount of divinylbenzene and the amount of diluent pass a critical value. The relationship between the two is given by Millar ${ }^{5}$ and Seidl, ${ }^{4}$ Figure $2 . \quad$ As is demonstrated in Figure 2, the degree of dilution influences the porosity of all types of copolymers. Therefore, experiments 01 to 04 were carried out in a constant volume of the organic phase. In view of the divinylbenzene content of $15 \%$ and an $f_{m}$ value of 0.43 , experiment 01 is expected to result in a porous resin (curve $2 \mathrm{~b}$ ) and experiment 04 , in a nonporous one (curve 3a). 


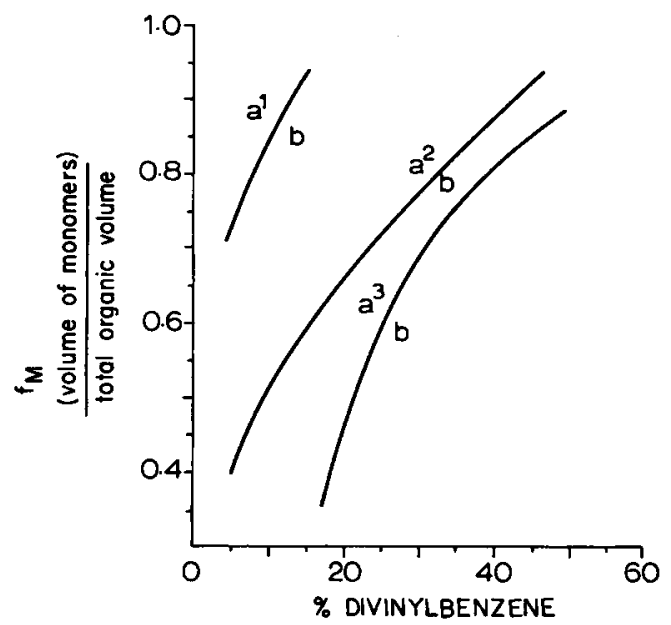

Fig. 2. $1=\mathrm{PM} ; 2=\mathrm{PP} ; 3=\mathrm{PS} ; \mathrm{a}=$ nonporous; $\mathrm{b}=$ porous.

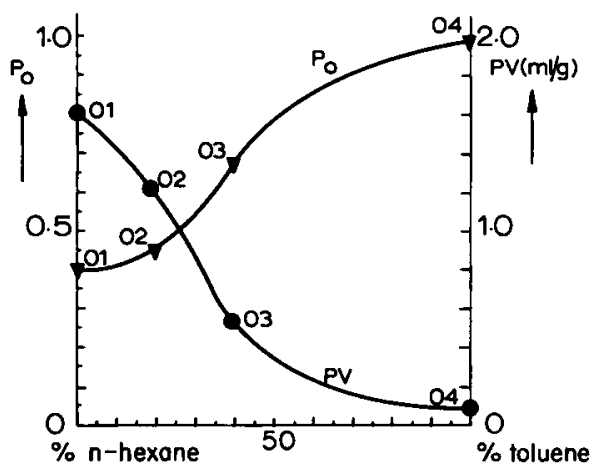

Fig. 3.

The effect of increasing the amount of solvent on the copolymer is clearly demonstrated by the apparent densities and the pore volumes in Table II and by its graphic presentation in Figure 3. The larger the amount of solvent, the smaller the pore volume; the resin then behaves more and more like a nonporous PS copolymer.

The method of changing a porous PP resin into a nonporous PMS copolymer by replacing the precipitator for a solvent is clearly of little interest. Therefore, we abandoned the constant volume of the organic phase and investigated the influence of a small increase of solvent on a PP copolymer with a fixed composition (experiments 0.5-0.8 and 09-12). Again, the effect is clearly demonstrated by the apparent densities and the pore volumes shown in Table II.

The pore spectrum of experiments 05 and 06 (Fig. 4) and the values of the average pore diameter (experiments 09-12) indicate that a small increase in solvent has a striking influence on the pore structure of the matrices. This gives us a tool to regulate the pore size distribution. A 


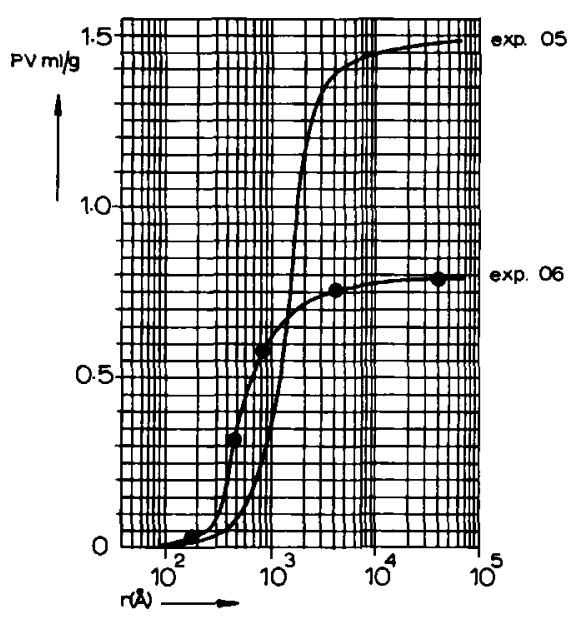

Fig. 4.

larger pore volume requires more nonsolvent; smaller pores and hence an enhanced internal surface are obtained by more solvent.

\section{PMS and PMP Resin}

Reaction temperature as well as the following parameters determine the porosity of these types of copolymers: (1) amount of diluent; (2) amount of divinylbenzene; (3) amount and kind of linear polymer; and (4) molecular weight of the linear polymer.

For a PM resin the effect of the parameters 2,3 , and 4 is described by Seidl. ${ }^{1}$ We investigated the first three parameters for the PMS matrices and the first, third and fourth for the PMP resins. Therefore, experiments 13-26 and 27-35 were carried out (Table III).

\section{PMS Resin}

Varying the amount of solvent and keeping the other parameters constant (experiments $13-16,17-20,21-23$ ) shows that increasing the amount of solvent increases the pore volume without changing the pore size distribution very much (Fig. 5).

Therefore, the solvent acts on the PMS matrix as an increasing amount of linear polymer on the PM matrix. Comparison of the experiment sets $(14,18,21),(15,19,22)$, and $(16,20,23)$ shows the influence of parameter 2 on the PMS copolymers. The role of the divinylbenzene content is similar to that for a PM copolymer. ${ }^{1}$ The sets $(23,24),(16,25)$, and $(20,26)$ demonstrate the role of parameter 3 . The enhanced amounts of linear polymer correspond with decreased apparent densities and increased pore volumes. This again is a parallel with the carlier investigations for PM copolymers. ${ }^{1}$

\section{PMP Resin}

The relation between precipitator concentration and pore structure is given by the results of experiments $27-29$, see Table III. Here, we meet 


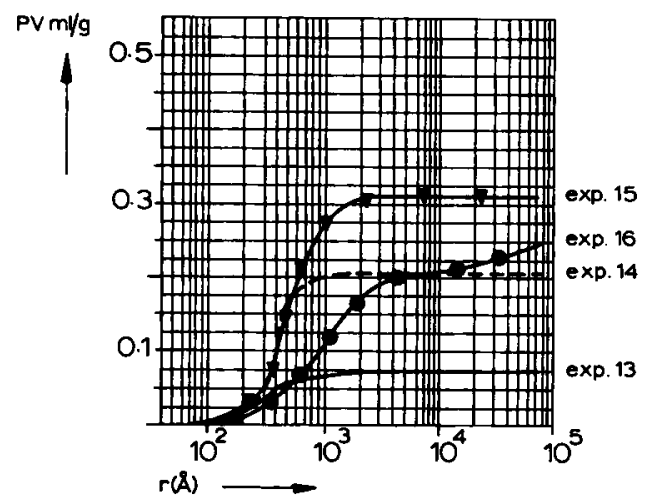

(a)

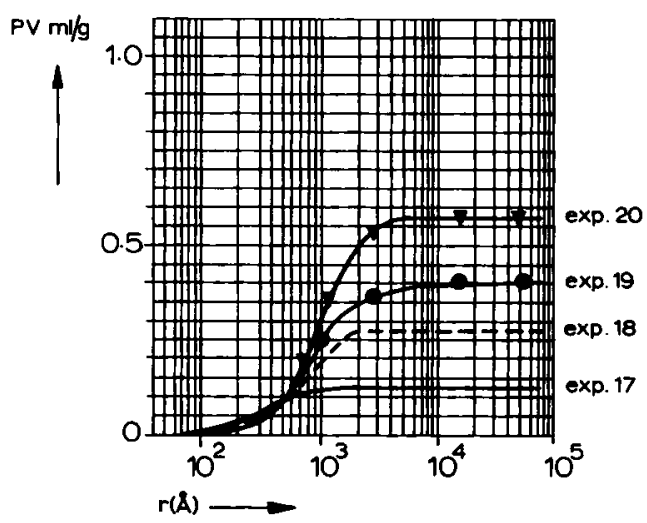

(b)

Fig. 5.

the main difference between the action of a solvent and the action of a nonsolvent on the styrene-divinylbenzene system. An increasing amount of solvent will increase the pore volume within certain limits, without changing the pore size distribution very much. Increase in concentration of nonsolvent will increase the pore volume and the pore size distribution. The latter property was already found in a PP copolymer, ${ }^{4}$ and made it possible to classify the PP resin on the appearance of the bead.

The role of the concentration of linear polymer is demonstrated by experiments 30-34. The pore spectra are given in Figure 6. Here, again, we have a tool to regulate rather accurately the desired pore size distribution. The effect of the molecular weight is treated in experiments 34 and 35 , see Figure 7.

\section{DISCUSSION}

\section{PPS Matrix}

An explanation of the effect of solvent on this type of copolymer can be given based on pore structure formation. This formation can be divided 


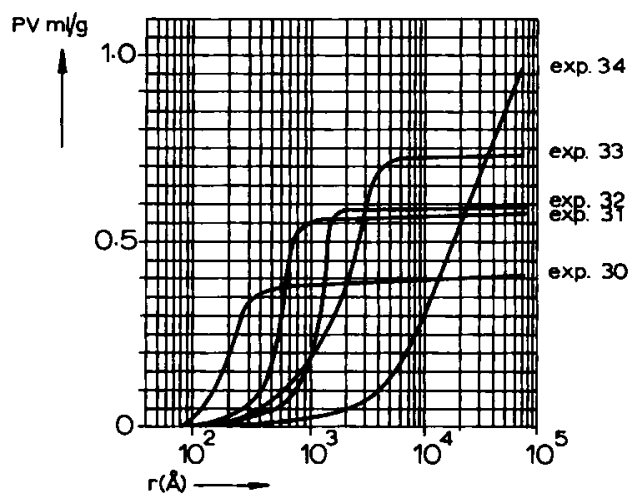

Fig. 6.

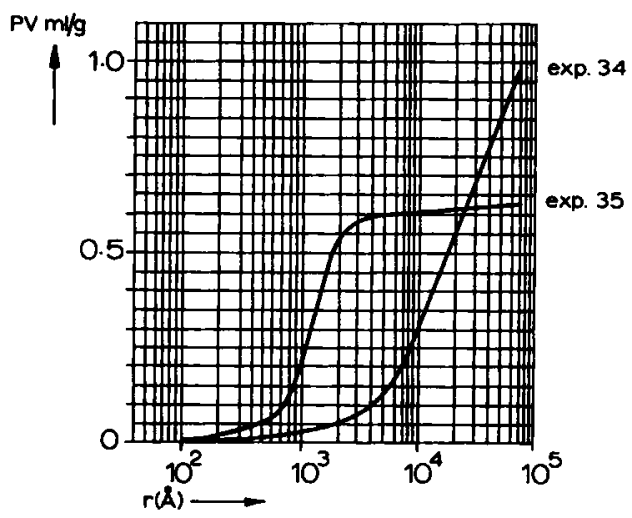

Fig. 7.

into three stages, ${ }^{11}$ characterized by (1) building of the microspheres, (2) agglomeration of microspheres, and (3) fixation of the agglomerates within the bead.

When the polymerization is carried out in the presence of a nonsolvating diluent (PP type) in the first stage of pore structure formation, a phase separation will occur, which is determined by the reaction temperature, the concentration of the crosslinking agent, and the concentration of the diluent. This separation gives a copolymer-rich phase and a diluent phase which is poor in copolymer. When the solvating monomers are converted. into copolymer, the polymer chains become less swollen and will become entangled by continuing polymerization. This yields the copolymer "nuclei" and their agglomerations.

During the second stage of pore structure formation, the entanglement continues. The agglomerations of the nuclei are forming the microspheres which for this type are expected to be rather compact. The microspheres are the smallest bodies that can be seen at the stereoscan photographs. The pores within such matrices are those among the microspheres (resulting in large transitional pores) and macrospheres. Hence, the pore volume of 


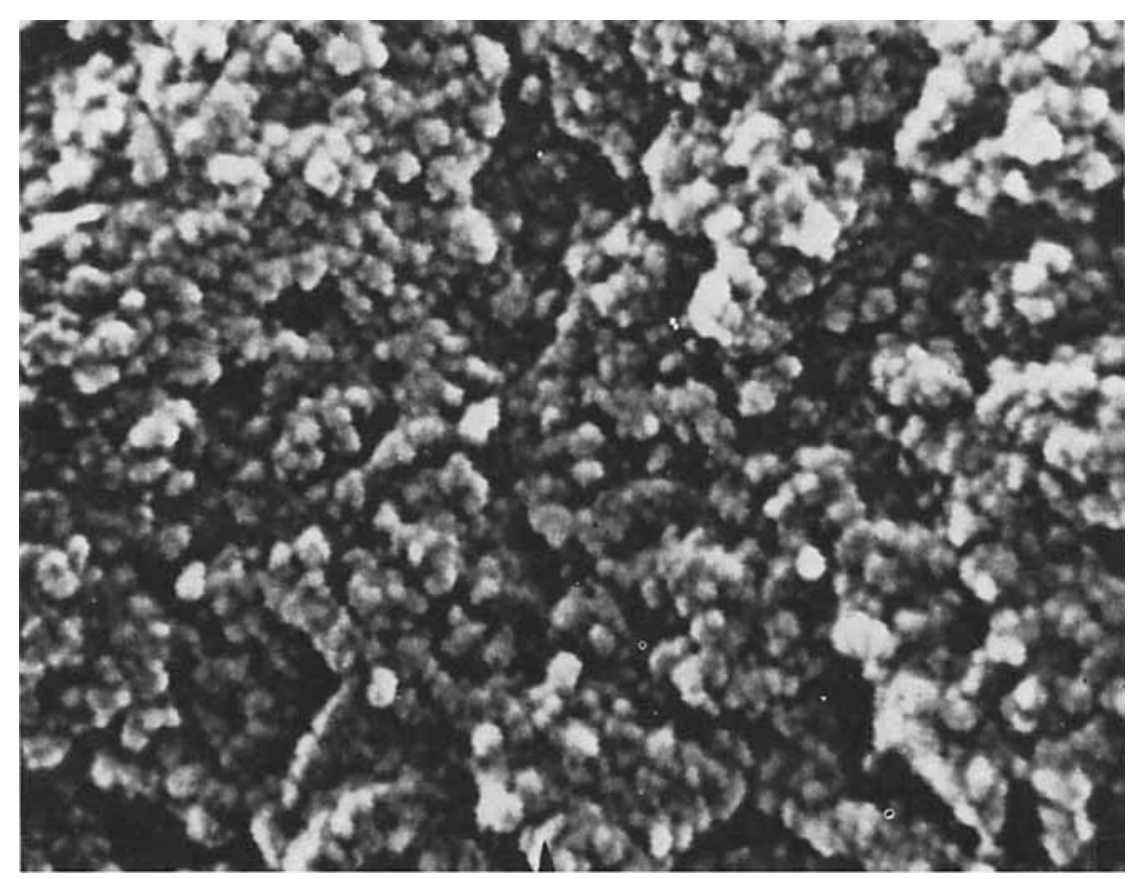

Fig. 8.

porous PP resins will be considerably large (Table II, Fig. 3). In the presence of a solvent in the reaction mixtures (PPS type), the polymer chains in the nuclei are less entangled as a consequence of the increased solvating character of the system. Also, during the second stage of pore structure formation, the agglomerations of the nuclei are more solvated compared to those formed in the absence of solvent. The microspheres are now less compact and will contain more pores of smaller sizes.

As the phase separation has a less drastic character, the pattern will be more regular. An electron micrograph, indeed, shows a regular formation of microspheres within the bead (Fig. 8). The small transitional pores and micropores within the microspheres have sizes that cannot be observed by electron microscopy. This means that compared to the PP type, the pores and the pore volume of PPS resin are smaller. This is confirmed by the pore spectra made by a mercury penetrometer (Fig. 3). It was demonstrated that better solvation enhances the internal surface. This can now be understood quite easily, because better solvation prevents the polymer chains from cohering, for the network is formed in an expanded state. This gives an increased microporosity that enhances the internal surface strikingly.

\section{PM Matrix}

We first consider the pore structure formation of a PM resin. For this type in the first stage, the polymer chains will be in a swollen state, because 


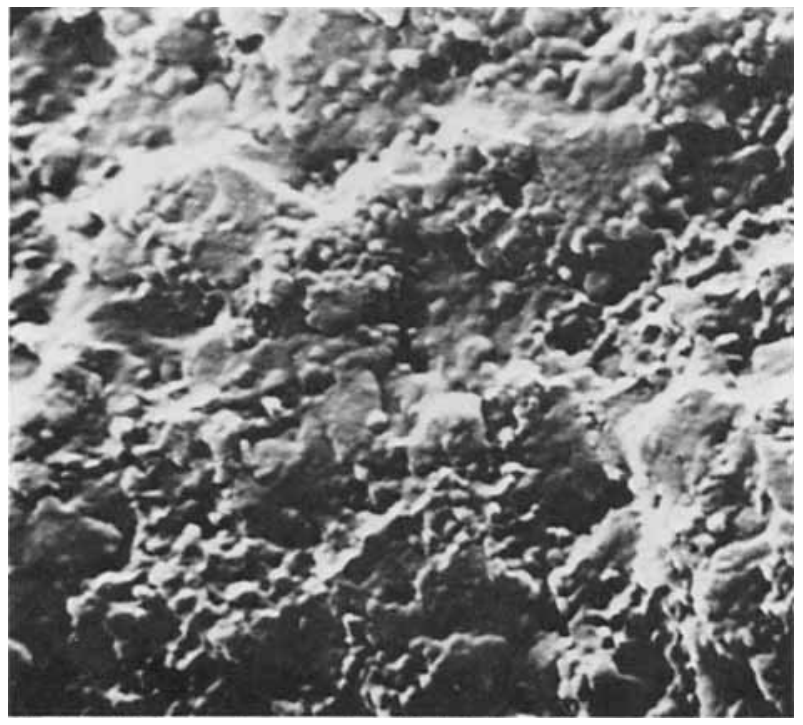

(a)

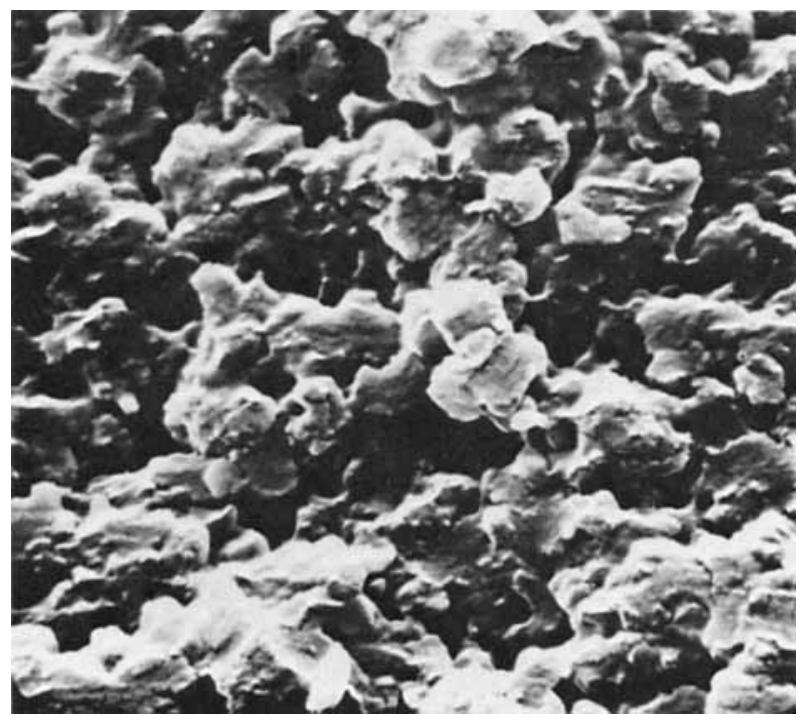

(b)

Fig. 9.

the monomers are acting as solvent. As the monomers are converted into copolymer, phase separation occurs between copolymer and linear polymer, which is strongly collapsing by loss of solvating monomer. The added linear polymer thus prevents the resulting network from having a complete gel structure.

Electron micrographs show quite a difference between the structure before and after the removal of linear polymer by extraction (Fig. 9). It must be noticed that extraction occurs by means of a solvating agent such 


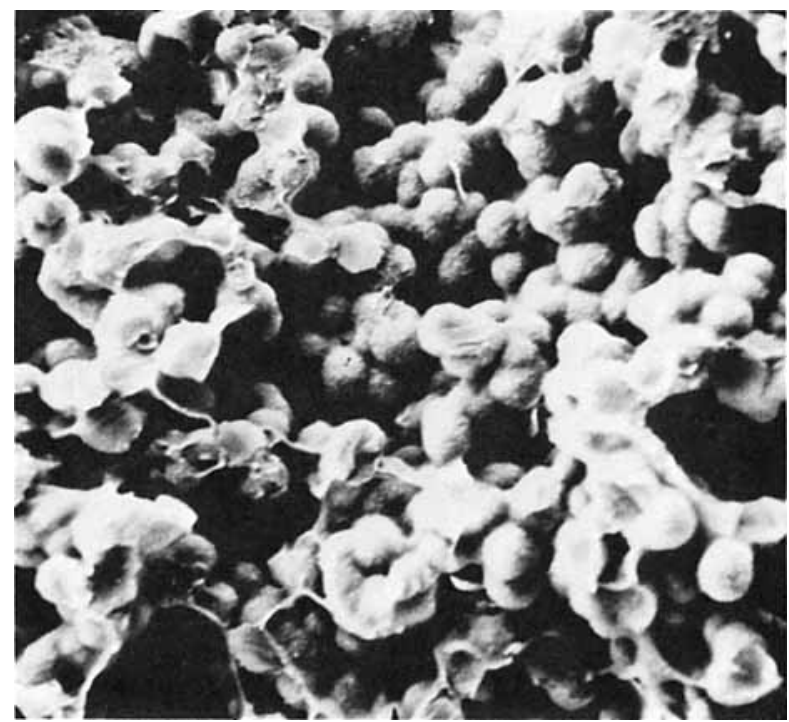

(a)

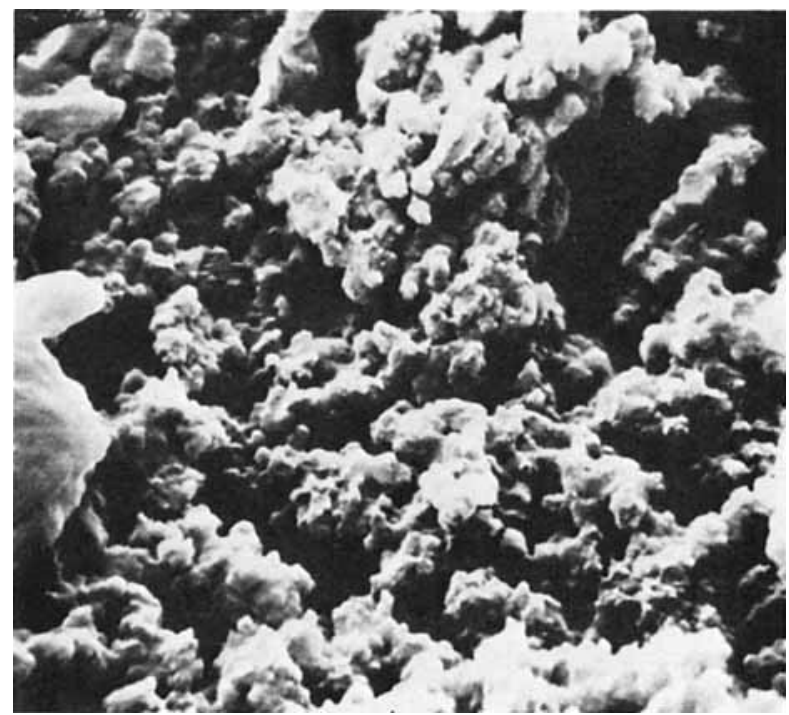

(b)

Fig. 10.

as dichloromethane or toluene, which agents have strong interaction with the polymer chains. As presented by Figure 9, the extracted PM resin (experiment 13) contains agglomerations with an average size of about $5000 \AA$ in dense packing.

\section{PMS Matrix}

The presence of a solvent during the suspension polymerization holds the network after being swollen in the monomers in a more expanded state. 
Compared to the PM resin, less collapse will occur, resulting in a higher porosity of network, characterized by a larger pore volume and a lower apparent density (experiments 13-20). The other experiments of this type have the same parameters as those described by Seidl. ${ }^{1}$

\section{PMP Matrix}

The model put forward also shows its validity for this type of resin. The nonsolvent added counteracts the monomer in solvating the polymer chains. After a certain time which is dependent on the concentration of the crosslinking agent, the diluent concentration, and the reaction temperature, (partial) phase separation occurs. Analogous to the mechanism of a PPS matrix pore structure formation, that of a PMP matrix results in large entangled nuclei. Agglomeration of the nuclei gives compact microspheres. Electron micrographs show particularly irregular structures, the microspheres having formed agglomerations of variable sizes (Fig. 10).

The network of experiment 34 shows microspheres varying from 1,000 to $5,000 \AA$, which are in their turn agglomerated to elements of $40,000 \AA$. The visible pores among the various agglomerations have sizes from 1,000 to $10,000 \AA$. The resin of experiment 35 differs from that of experiment 34 only in one respect: the molecular weight of the linear polymer used by the synthesis is 140,000 instead of 33,000 , which results in extremely large sizes both of the microspheres and of the pores. The agglomerated microspheres have sizes varying from 30,000 to $40,000 \AA$. The visible pores have extremely large sizes up to $150,000 \AA$, which is confirmed by the pore spectrum made by a mercury penetrometer (Fig. 7).

This work originated in the fruitful discussions with Drs. J. Cley and E. Bijleveld of A.C. Division Ketjen, Amsterdam. The authors also gratefully acknowledge AKZO Corporate Research, Arnhem, for making the stereoscan photographs and the A.C. Resear ch Laboratory, Amsterdam, for the B.E.T. determinations. The authors wish to thank Miss H. Hilgen, Mrs. E. Haas, and Miss H. von Pickartz of ACZ Division, Hengelo, for their help.

\section{References}

1. J. Seidl, J. Malinsky, K. Dusek, and W. Heitz, Advan. Polym. Sci., 5, 113 (1967).

2. J. R. Millar, D. C. Smith, W. E. Marr, and T. R. E. Kressman, J. Chem. Soc., 218 (1963).

3. A. S. Tevline and S. F. Sadova, Zh. Prikl. Khim., 38, 1643 (1965).

4. J. Seidl, Dissertation, Institute of Macromol. Chem-Czechoslovak Acad. of Science, Prague, Czechoslovakia, 1964.

5. J. R. Millar, D. C. Smith, and T. R. E. Kressman, J. Chem. Soc., 304 (1965).

6. E. Bortel, Przemy Chem., 44, 255 (1965).

7. I. M. Abrams, Ind. Eng. Chem., 48, 1469 (1956).

8. J. Seidl and J. Malinsky, Chem. Prumysl, 13, 100 (1963).

9. K. Dusek, J. Seidl, and J. Malinsky, Chem. Prumysl, 13, 662 (1963).

10. K. A. Kun and R. Kunin, J. Polym. Sci. C, 16, 1457 (1967).

11. K. A. Kun and R. Kunin, J. Polym. Sei. A-1, 7, 2689 (1968).

12. S. Brunauer, Physical Adsorption, Vol. 1, Princeton University Press, Princeton, N.J., 1943.

Received January 12, 1973 